\title{
PURIFICACIÓN DE LA FRACCIÓN ANTIGÉNICA 27-28 KDA A PARTIR DEL ANTÍGENO METABÓLICO SECRETADO- EXCRETADO DE Fasciola hepatica
}

\author{
Isidro Antitupa ${ }^{1, a}$, William Quispe ${ }^{1, a}$, Jhon Mayo ${ }^{1, a}$, Fanny Valverde ${ }^{1, b}$, Elizabeth Sanchez ${ }^{1, a, c, d}$
}

\begin{abstract}
RESUMEN
En el presente estudio, las fracciones antigénicas de 27-28 KDa de Fasciola hepatica fueron purificadas por cromatografía de exclusión molecular para su aplicación en el diagnóstico de la fascioliasis humana. Se obtuvieron antígenos de excreción y secreción a partir de fasciolas adultas vivas obtenida de hígado de ovino y bovino, y cultivados en medio mínimo esencial. La reactividad y eficacia del antígeno purificado fueron evaluadas por la prueba de inmunoblot empleando cuatro sueros con fascioliasis humana; cuatro sueros con otras parasitosis, y dos sueros negativos. Se concluye que las fracciones antigénicas purificadas no presentan reacción cruzada con otras parasitosis, por inmunoblot, por lo que se considera a las proteínas purificadas como potenciales candidatas a ser utilizadas para el diagnóstico de fascioliasis humana.
\end{abstract}

Palabras clave: Fasciola hepatica; Purificación; Cromatografía (fuente: DeCS BIREME).

\section{PURIFICATION OF ANTIGENIC FRACTION 27-28 KDA FROM THE METABOLIC ANTIGEN FROM METABOLIC SECRETED-EXCRETED FROM Fasciola hepatica}

\begin{abstract}
Antigenic fractions of 27-28 kDa from Fasciola hepatica were purified by size-exclusion chromatography for use in the diagnosis of human fasciolosis. Excretion and secretion antigens were obtained from living adult flukes collected from sheep and cattle liver, and cultured in minimum essential medium. The reactivity of the purified antigen and efficacy were assessed by immunoblot test using four sera with human fascioliasis; four sera with other parasites, and two negative sera. We conclude that the purified antigenic fractions do not cross-react with other parasites by immunoblot. Therefore, purified proteins are considered as potential candidates to be used for the diagnosis of human fascioliasis.
\end{abstract}

Key words: Fasciola hepatica; Purification; Chromatography (source: MeSH NLM).

\section{INTRODUCCIÓN}

Los antígenos metabólicos de excreción-secreción de Fasciola hepatica (AgMFhE/S total), han sido reportados como potenciales antígenos de importancia inmunológica debido a su importante rol en inducir la formación de anticuerpos. Los productos de AgMFhE/S total, tienen en su composición proteasas que participan en la nutrición y son consideradas factores importantes de virulencia así como en la evasión de la respuesta inmune del parásito y en la invasión de tejidos del hospedero ${ }^{(1,2)}$.

Algunos análisis electroforéticos y bioquímicos del antígeno total AgMFhE/S de F. hepatica, mostraron 31 bandas de naturaleza polipeptídica en rango de 10-116 KDa, dentro de los cuales destacan un grupo de siete polipéptidos entre 66-116 KDa; un segundo grupo de nueve polipéptidos entre $60-31 \mathrm{KDa}$, un tercero con seis polipéptidos entre 29-17 KDa, y una agrupación de nueve polipéptidos entre $10-15 \mathrm{KDa}{ }^{(3,4)}$. Estos antígenos son utilizados en pruebas serológicas con enfoque diagnóstico, con valores variables de sensibilidad y especificidad $(5,6)$. Se conocen cuatro bandas de diagnóstico específicas $(8,12,15$ y $24 \mathrm{kDa})$ en pacientes infectados con $F$. hepatica ${ }^{(6)}$. En Perú se ha evaluado la eficacia del inmunoblot para inmunoglobulina G (IgG) utilizando AgMFhE/S totales, encontrándose que la bandas 17 y $23 \mathrm{KDa}$ son altamente sensibles y específicas ${ }^{(5)}$. A la vez, se encontraron reacciones cruzadas con otras parasitosis como paragonimiasis, amebiasis y enfermedades crónicas hepáticas ${ }^{(5,16)}$.

\footnotetext{
Laboratorio de Zoonosis Parasitaria, Instituto Nacional de Salud. Lima, Perú.

Biólogo; ${ }^{\mathrm{b}}$ Técnico de Laboratorio; ${ }^{\mathrm{C}}$ Magíster en Biología Parasitaria; ${ }^{\mathrm{d}}$ Doctor en Ciencias.

Recibido: 14-01-14 Aprobado: 28-05-14
}

Citar como: Antitupa I, Quispe W, Mayo J, Valverde F, Sanchez E. Purificación de la fracción antigénica 27-28 KDa a partir del antígeno metabólico secretadoexcretado de Fasciola hepatica. Rev Peru Med Exp Salud Publica. 2014;31(2):288-91. 
También fueron evaluados antígenos purificados que mostraron valores de especificidad y sensibilidad entre 94,5 y $100 \%$, con fracciones antigénicas purificadas de pesos moleculares de $14 \mathrm{KDa}$ y $24-29 \mathrm{KDa}{ }^{(9,17)}$.

Se ha observado que un componente antigénico del producto metabólico AgMFhE/S total del parásito adulto con una masa molecular aproximada de $27 \mathrm{KDa}$ podría tener importancia inmunodiagnóstica hasta con $100 \%$ de sensibilidad y $98 \%$ de especificidad. ${ }^{(7)}$. La purificación parcial de AgMFhE/S total de F. hepatica utilizando Sephadex G-200 elimina gran parte reactividad cruzada con sueros de fascioliasis ${ }^{(3,8)}$.

El propósito del presente estudio es purificar una fracción antigénica (27-28 kDa) de F. hepatica mediante cromatografía de exclusión molecular para su aplicación en el diagnóstico de la fascioliasis humana.

\section{EL ESTUDIO}

\section{ANTÍGENO TOTAL}

El antígeno total AgMFhE/S fue obtenido a partir de parásitos adultos obtenidos de hígado de ovinos y bovinos naturalmente infectados. Los parásitos fueron lavados con solución fosfato salino (PBS) $0,01 \mathrm{M}$ a $\mathrm{pH}=7,2$. Estos, a su vez, fueron cultivados en $100 \mathrm{~mL}$ de medio mínimo esencial (MEM) (GIBCO ${ }^{\mathrm{TM}}$ ), enriquecido con bicarbonato de sodio a $\mathrm{pH}=7,2$ a $37{ }^{\circ} \mathrm{C}$ (cincuenta parásitos por placa). El sobrenadante del cultivo colectados cada $4 \mathrm{~h}$, durante $16 \mathrm{~h}$, fue dializado en membrana de diálisis de 12 -14KD (Spectra/Por 1), en agitación contra 100 volúmenes de agua destilada. Finalmente, el dializado fue centrifugado $10000 \times \mathrm{g}$ durante $30 \mathrm{~min}$ a $4{ }^{\circ} \mathrm{C}$. La concentración de proteínas del antígeno fue determinada por el método de Lowry.

\section{ELECTROFORESIS SDS-PAGE}

El análisis del perfil proteico del AgMFhE/S total y purificado se realizó por SDS-PAGE en gel de poliacrilamida al $12 \%$ en un equipo Mini-Protean II (BIO-RAD) a una diferencia potencial de $160 \mathrm{~V}$. Se utilizó $8 \mu \mathrm{L}$ de antígeno en concentración de 1,15 $\mu \mathrm{g} / \mu \mathrm{L}$. El gel fue coloreado con nitrato de plata.

\section{ANTÍGENO PURIFICADO}

La purificación de AgMFhE/S se realizó por cromatografía de exclusión molecular (sephadex G-100), equilibrado con buffer de equilibrio $\mathrm{pH}=7,2\left(0,01 \mathrm{M} \mathrm{NaH}_{2} \mathrm{PO}_{4}, 0,01 \mathrm{M}\right.$ $\mathrm{Na}_{2} \mathrm{HPO}_{4}$ ). Finalmente, se usó $2 \mathrm{~mL}$ de AgMFhE/S [1,15 $\mu \mathrm{g} / \mu \mathrm{L}]$ alicuotado después de la diálisis. La elución de los antígenos se realizó con $\mathrm{NaCl} 1 \mathrm{M}$; se colectó trece fracciones en tubos de 1,5 mL; luego se determinó la concentración proteica de cada fracción y, finalmente, se realizó una electroforesis SDS-PAGE al 12\%. Las fracciones que contenían las proteínas purificadas se transfirieron a membranas de nitrocelulosa (BIO-RAD) por electrotransferencia a voltaje constante de $55 \mathrm{~V}$ por $75 \mathrm{~min}$.

\section{REACCIÓN INMUNOENZIMATICA}

La selección de los sueros en la seroteca se hizo de acuerdo con los siguientes criterios: examen parasitológico (heces) y serológico (ELISA). Se tomaron cuatro sueros de fascioliasis, un suero de hidatidosis, un suero de cisticercosis, un suero de paragonimiasis, un suero de Blastocystis hominis y dos sueros negativos, para evaluar su reactividad antígeno-anticuerpo mediante la técnica de Western Blot con las tiras reactivas de AgMFhE/S total y AgMFhE/S purificados que contienen las fracciones 27-28 KDa.

\section{HALLAZGOS}

La concentración del AgMFhE/S total fue de 2,07 ug/uL; en la caracterización proteica en geles SDS-PAGE al $17 \%$ se observó la presencia de diez bandas de pesos moleculares aproximados: 64,$1 ; 28,3 ; 27,4 ; 20,4 ; 17,1$; 14,$1 ; 8,8 ; 6,5 ; 5,9$ y 4,1 KDa visualizándose la fracción de interés a purificar; estos pesos moleculares aproximados fueron determinados con un coeficiente de confiabilidad de $95 \%$, comparando la movilidad electroforética (Rf) de la proteína de peso molecular desconocido con el del MPM (BIO-RAD) (datos no mostrados).

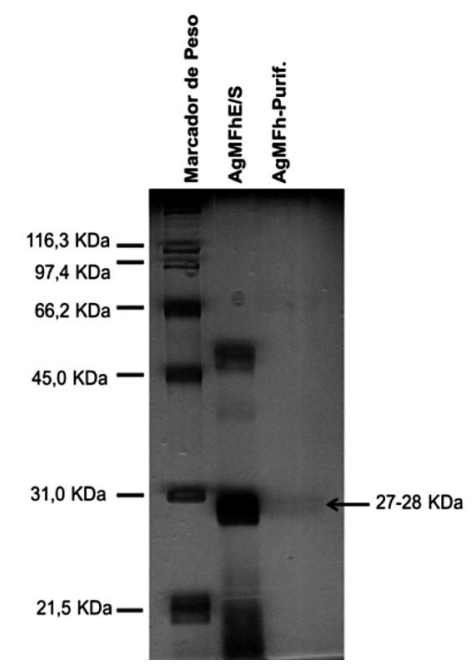

Figura 1. Electroforesis en SDS-PAGE al $12 \%$ de AgMFhE/S purificados por cromatografía de exclusión molecular. Se puede observar que la flecha indica la banda de 27-28 kDa purificado en una matriz de Sephadex G-100. Linea A, MPM (BIORAD); línea $B, 10 \mu \mathrm{L}$ de AgMFhE/S total [2,73 ug/uL]; línea $\mathrm{C}, 10 \mu \mathrm{L}$ de fracción purificada de AgMFhE/S [0,93 ug/uL] 


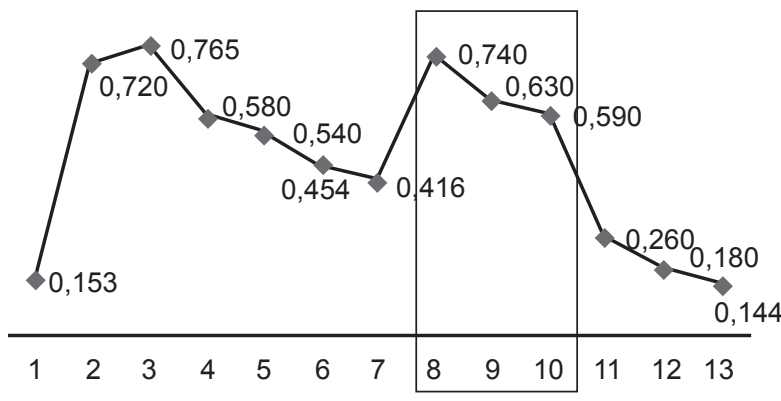

Figura 2. Cuantificación de las fracciones de AgMFhE/S purificadas por cromatografía de exclusión molecular en una matriz de sephadex G-100. Las fracciones 8,9 y 10 indican las fracciones donde caen las bandas de interés (27-28 KDa); en cada fracción se puede observar las diferentes concentraciones de antígeno en ug/uL

Mediante cromatografía por exclusión molecular utilizando sephadex G-100, que es una técnica que permite separar moléculas en función de su tamaño molecular, se purificó bandas de importancia diagnóstica de pesos moleculares aproximados entre 27-28 KDa a partir de AgMFhE/S totales (Figura 1); a la vez, estas fracciones purificadas caen en las alícuotas 8,9 y 10 con concentraciones de 0,740 ug/ uL; 0,630 ug/uL y 0,590 ug/uL respectivamente (Figura 2), los picos más altos se grafican cuando las alícuotas 8,9 y 10 contienen las proteínas de interés purificados.

Una vez obtenidas las fracciones purificadas, se probó su utilidad en el diagnóstico de fascioliasis humana, para esto se procedió a realizar una prueba de Western Blot

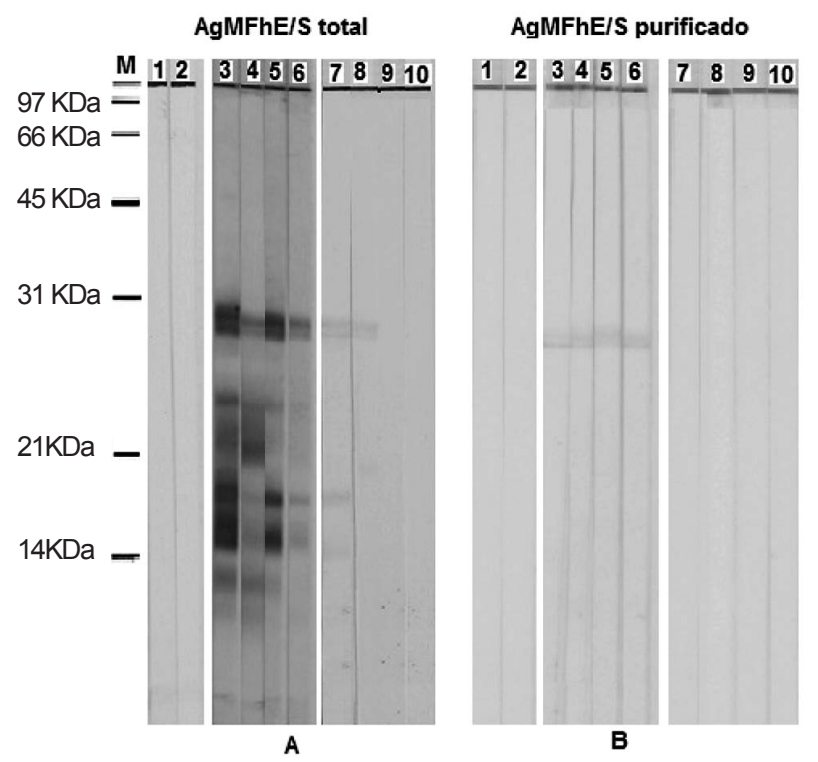

Figura 3. Prueba de Western Blot utilizando las fracciones totales $(A)$ y purificados $(B)$ para el diagnóstico de $F$. hepatica en humanos: M: MPM (BIO-RAD); 1 y 2: sueros control negativo; $3,4,5$ y 6 : sueros positivos para fascioliasis humana; $7,8,9$ y 10: sueros de otras parasitosis. La flecha indica la fracción antigénica purificada $27-28 \mathrm{KDa}$. utilizando estas fracciones purificadas, luego se evaluó con sueros de pacientes con fascioliasis confirmados, con sueros controles negativos y sueros con otras parasitosis. A la vez, se comparó con tiras producidas AgMFhE/S totales (Figura 3).

En la reacción inmunoenzimática se observó que los AgMFhE/S totales mostraron bandas reactivas de pesos moleculares aproximados de $8,8 \mathrm{KDa}$ hasta 27 $28 \mathrm{KDa}(8.8,14.2,17.1,20.4,27-28 \mathrm{KDa})$ con sueros de pacientes con fascioliasis humana confirmadas mediante examen coprológico y serológico, detectando las cuatro muestras como positivas. En cambio, las tiras reactivas que contienen AgMFhE/S total, que fueron incubadas con sueros de otras parasitosis, hacen reacción cruzada a Blastocystis hominis y Paragonimus spp, con la presencia de bandas de peso molecular 20,4; 27-28 KDa (Figura 3A), mas no con sueros de pacientes con cisticercosis e hidatidosis.

En cambio los AgMFhE/S purificados no presentaron reacciones cruzadas; también se detecta las cuatro muestras de pacientes con fascioliasis humana confirmada, donde se puede observar la presencia de la banda 27-28 KDa (Figura 3B). Esto demuestra la especificidad y sensibilidad de esta fracción purificada y su potencial uso en el diagnóstico de fascioliasis humana por Western Blot y en otras pruebas de serología.

\section{DISCUSIÓN}

La concentración de AgMFhE/S purificado desde AgMFhE/S total, es directamente proporcional, es decir cuanto mayor es la concentración de AgMFhE/S total empleado para la purificación, se obtiene mayor concentración de AgMFhE/S purificado. Por ejemplo, de una alícuota de concentración de 2,73 ug/uL del AgMFhE/S total se obtiene $0,90 \mathrm{ug} / \mathrm{uL}$ del AgMFhE/S purificado.

Los AgMFhE/S totales de $F$. hepatica son proteinasas que se encuentran en el exterior del parásito, como resultado de regurgitación (10), así como los AgMFhE/S totales obtenidos en el presente trabajo. Por lo tanto se puede decir que la fracción molecular purificada (27-28 KDa) es de esta misma naturaleza, que puede estar implicada en la digestión extracelular de las células intestinales ${ }^{(11)}$. También se observó que cuando los parásitos inmaduros y maduros de $F$. hepatica, se mantenían en cultivo durante $16 \mathrm{~h}$ se liberaban metabolitos como producto de la secreción - excreción tegumentaria y alimenticia, que influyen en la migración del parásito. Como se ha evaluado en otros trabajos estas enzimas fueron clasificadas como proteinasas de cisteína, divididas en dos grupos (grupo 1: 60-90 KDa y 
grupo 2: 27,5 a $50 \mathrm{KDa})^{(12)}$, esto se puede comparar con los resultados obtenidos, en donde la fracción purificada tiene un peso molecular aproximado de 27-28 KDa.

Las fracciones de pesos moleculares aproximados $27 \mathrm{KDa}$ y 25-26 KDa son de naturaleza proteinasa como catepsina L-like, según el análisis de las secuencias N-terminal, se trata de una de las proteínas más importantes excretadas y secretadas por $F$. hepatica in vitro y son las moléculas predominantes en un $80 \%$, también se pudo demostrar por microscopía que se encuentran en vesículas a nivel de las células epiteliales del intestino de F. hepatica $(13,14)$. Si solo nos basaríamos en el peso molecular de la fracción purificada en este trabajo, se podría decir que se trata de la misma proteinasa que tiene mucha importancia en el inmunodiagnóstico de la fascioliasis humana; para demostrar esto es recomendable realizar un trabajo de caracterización molecular y bioquímica de esta fracción; ya que esta proteinasa, catepsina L-like, se usa en otros países en pruebas de ELISA y Western Blot, obteniéndose buenos resultados y que ahora podrían ser utilizados como una proteína de importancia inmunodiagnóstica (15-17). Esto es correlativo con este trabajo, puesto que de las cuatro muestras evaluadas para fascioliasis humana confirmadas, detectaron todas y discriminaron muy bien entre las muestras con otras parasitosis; por eso, es recomendable hacer un siguiente trabajo de evaluación de esta proteína purificada con un panel de sueros más grande, además de evaluar su sensibilidad y especificidad. Por lo tanto, se puede concluir que se logró purificar la fracción de peso molecular aproximado de $27-28 \mathrm{KDa}$, y que es un potencial candidato para el inmunodiagnóstico de fascioliasis humana.

Contribuciones de autoría: FVT y JMA han participado en la recolección del hígado infectado con los parásitos adultos y en la preparación de los materiales que se usaron durante el desarrollo del trabajo. JMA y WQP han participado en la obtención de antígenos totales y control de calidad de los antígenos. IAJ, ha participado en la purificación del antígeno WQP, IAJ y ESR participaron en la redacción del artículo así como de la revisión crítica y aprobación de la versión final.

Fuentes de financiamiento: Instituto Nacional de Salud de Perú.

Conflictos de interés: los autores declaran no tener conflictos de interés.

\section{REFERENCIAS BIBLIOGRÁFICAS}

1. Berasain P, Goñi F, McGonigle $S$, Dowd A, Dalton JP, Frangione B, et al. Proteinases secreted by Fasciola hepatica degrade extracellular matrix and basement membrane components. J Parasitol. 1997 Feb;83(1):1-5.

2. Berasain P, Carmona C, Frangione B, Dalton JP, Goñi F. Fasciola hepatica: parasite-secreted proteinases degrade all human IgG subclasses: determination of the specific cleavage sites and identification of the immunoglobulin fragments produced. Exp Parasitol. 2000 Feb;94(2):99-110.

3. Santiago N, Hillyer GV. Isolation of potential serodiagnostic Fasciola hepatica antigens by electroelution from polyacrylamide gels. Am J Trop Med Hyg. 1986 Nov;35(6):1210-7.

4. El-Rahimy HH, Mahgoub AM, ElGebaly NS, Mousa WM, Antably AS. Molecular, biochemical, and morphometric characterization of Fasciola species potentially causing zoonotic disease in Egypt. Parasitol Res. 2012 Sep;111(3):1103-11. doi: 10.1007/ s00436-012-2938-2.

5. EscalanteH,Davelois K, Ortiz P, Rodríguez H, Díaz E, Jara C. Estandarización de la técnica de Western blot para el diagnóstico de la fasciolosis humana utilizando antígenos de excreción-secreción de Fasciola hepática. Rev Peru Med Exp Salud Publica. 2011 Jul-Set;28(3):454-61.
6. De Almeida MA, Ferreira MB, Planchart S, Terashima A, Maco V, Marcos L, et al. Preliminary antigenic characterisation of an adult worm vomit preparation of Fasciola hepatica by infected human sera. Rev Inst Med Trop Sao Paulo. 2007 JanFeb;49(1):31-5.

7. Intapan PM, Maleewong W, Wongkham $\mathrm{C}$, Tomanakarn $\mathrm{K}$, Ieamviteevanich $\mathrm{K}$, Pipitgool V, et al. Excretory-secretory antigenic components of adult Fasciola gigantica recognized by infected human sera. Southeast Asian J Trop Med Public Health. 1998 Sep;29(3):579-83.

8. Youssef FG, Mansour NS. A purified Fasciola gigantica worm antigen for the serodiagnosis of human fascioliasis. Trans R Soc Trop Med Hyg. 1991 JulAug;85(4):535-7.

9. Silva $\mathrm{M}$, Gorman $\mathrm{T}$, Alcaíno $\mathrm{H}$. Inmunodiagnóstico de fasciolosis humana y ovina empleando una fracción de 24-29 $\mathrm{kDa}$ de Fasciola hepatica obtenida mediante inmunoadsorción. Parasitol Latinoam. 2005 Jun;60(1-2):38-42.

10. Locatelli A, Beretta C. Detection of aminoacid $\mathrm{N}$ in saline or serum media incubated with Fasciola hepatica and proteolytic activity exerted by liver fluke in vitro. Arch Vet Ital. 1969 Oct 31;20(5):385-91.

11. Howell MJ. Localization of proteolytic activity in Fasciola hepatica.J Parasitol. 1973 Jun;59(3):454-6.
12. Dalton JP, Heffernan M. Thiol proteases released in vitro by Fasciola hepatica. Mol Biochem Parasitol. 1989 Jun 15;35(2):161-6.

13. Smith AM, Dowd AJ, McGonigle S, Keegan PS, Brennan G, Trudgett A, et al. Purification of a cathepsin L-like proteinase secreted by adult Fasciola hepatica. Mol Biochem Parasitol. 1993 Nov;62(1):1-8.

14. McGinty A, Moore M, Halton DW, Walker B. Characterization of the cysteine proteinases of the common liver fluke Fasciola hepatica using novel, active-site directed affinity labels. Parasitology. 1993 Jun;106 ( Pt 5):487-93.

15. Yamasaki $H$, Mineki R, Murayama K, Ito A, Aoki T. Characterisation and expression of the Fasciola gigantica cathepsin L gene. Int J Parasitol. 2002 Jul;32(8):1031-42.

16. Gorman T, Sanchez R, Fredes F, Alcaino H. Inmunodiagnóstico de fasciolosis bovina mediante ELISA y Western Blot. Parasitol Dia. 1998 Jan;22(1-2):16-22.

17. Fredes F, Gorman T, Silva M, Alcaino H. Evaluación diagnóstica de fracciones cromatográficas de Fasciola hepatica mediante Western Blot y ELISA en animales infectados. Arch Med Vet. 1997;29(2):283-94.

Correspondencia: Isidro Antitupa Janampa

Dirección: Calle Cápac Yupanqui 1400 - Jesus

Maria, Lima, Perú.

Teléfono: (511) 7481111 Anexo 2154

Correo electrónico:ant.isidro@gmail.com 